\title{
GIANNI ARIOLI
}

\section{FILIPPO GAZZOLA}

\section{Weak solutions of quasilinear elliptic PDE's at resonance}

Annales de la faculté des sciences de Toulouse $6^{e}$ série, tome $6, n^{\circ} 4$ (1997), p. 573-589

<http://www.numdam.org/item?id=AFST_1997_6_6_4_573_0>

(C) Université Paul Sabatier, 1997, tous droits réservés.

L'accès aux archives de la revue «Annales de la faculté des sciences de Toulouse » (http://picard.ups-tlse.fr/ annales/) implique l'accord avec les conditions générales d'utilisation (http://www.numdam.org/conditions). Toute utilisation commerciale ou impression systématique est constitutive d'une infraction pénale. Toute copie ou impression de ce fichier doit contenir la présente mention de copyright.

\section{Numdam}

Article numérisé dans le cadre du programme Numérisation de documents anciens mathématiques http://www.numdam.org/ 


\title{
Weak solutions of quasilinear elliptic PDE's at resonance ${ }^{(*)}$
}

\author{
Gianni ARIoli ${ }^{(1)}$ and Filippo Gazzola ${ }^{(1)}$
}

RÉsumÉ. - Nous étudions une certaine classe d'EDP quasi linéaires variationnelles avec résonance à l'infini en utilisant une théorie des points critiques pour fonctionnelles irrégulières. Nous démontrons l'existence d'une solution dans le cas général et un résultat de multiplicité lorsque l'équation est invariante par rapport à une action du groupe $\mathbb{Z}_{2}$.

ABstract. - We study a class of variational quasilinear elliptic PDE's with resonance at infinity by means of a nonsmooth critical point theory. We prove the existence of a solution in the general case and a multiplicity result when the equation is invariant with respect to a $\mathbb{Z}_{2}$-action.

\section{Introduction}

Let $\Omega$ be an open bounded subset of $\mathbb{R}^{n}$ with $n \geq 3$; in this paper we prove the existence of functions $u \in H_{0}^{1}$ satisfying in a weak sense the following equation

$$
-\sum_{i, j=1}^{n} \mathrm{D}_{j}\left(a_{i j}(x, u) \mathrm{D}_{i} u\right)+\frac{1}{2} \sum_{i, j=1}^{n} \frac{\partial a_{i j}}{\partial s}(x, u) \mathrm{D}_{i} u \mathrm{D}_{j} u=b(x) u+g(x, u) .
$$

We assume the ellipticity condition and the "usual" semipositivity condition (see [3]) on the matrix $\left[s \partial a_{i j}(x, s) / \partial s\right]$ to be fulfilled:

$$
\left\{\begin{array}{l}
\exists \nu>0 \text { such that for a.e. } x \in \Omega, \forall s \in \mathbb{R}, \forall \xi \in \mathbb{R}^{n}, \\
\sum_{i, j=1}^{n} a_{i j}(x, s) \xi_{i} \xi_{j} \geq \nu|\xi|^{2}
\end{array}\right.
$$

(*) Reçu le 20 septembre 1995

(1) Dipartimento di Scienze T.A, via Cavour 84, I-15100 Alessandria (Italy) 


$$
\begin{gathered}
\text { Gianni Arioli and Filippo Gazzola } \\
\left\{\begin{array}{l}
s \sum_{i, j=1}^{n} \frac{\partial a_{i j}}{\partial s}(x, s) \xi_{i} \xi_{j} \geq 0 \\
\text { for a.e. } x \in \Omega, \forall s \in \mathbb{R}, \forall \xi \in \mathbb{R}^{n}
\end{array}\right.
\end{gathered}
$$

We require the coefficients $a_{i j}(x, u)$ to satisfy

Assume that

$$
\left\{\begin{array}{l}
a_{i j} \equiv a_{j i} \\
a_{i j}(x, s), \quad \frac{\partial a_{i j}}{\partial s}(x, s) \in L^{\infty}(\Omega \times \mathbb{R}, \mathbb{R}) \\
a_{i j}(x, \cdot) \in C^{1}(\mathbb{R}) \text { for a.e. } x \in \Omega \\
\lim _{|s| \rightarrow \infty} a_{i j}(x, s)=A_{i j}(x) .
\end{array}\right.
$$

$$
b \in L^{n / 2}(\Omega)
$$

denote by $G(x, s)=\int_{0}^{s} g(x, t) \mathrm{d} t$ and assume that there exist $\alpha \in$ $L^{2 n /(n+2)}(\Omega), \beta \in L^{n / 2}(\Omega)$ and $\gamma \in L^{1}(\Omega)$ such that

$$
\begin{cases}g: \Omega \times \mathbb{R} \rightarrow \mathbb{R} & \text { is a Carathéodory function } \\ |g(x, s)| \leq \alpha(x)+\beta(x)|s| & \forall s \in \mathbb{R} \text { and for a.e. } x \in \Omega \\ \lim _{|s| \rightarrow \infty} \frac{g(x, s)}{s}=0 & \text { uniformly w.r.t. } x \in \Omega \\ G(x, s) \rightarrow+\infty & \text { if }|s| \rightarrow \infty \text { for a.e. } x \in \Omega \\ 2 G(x, s) \rightarrow s g(x, s) \rightarrow+\infty & \text { if }|s| \rightarrow \infty \text { for a.e. } x \in \Omega \\ 2 G(x, s)-s g(x, s) \geq \gamma(x) & \text { for a.e. } x \in \Omega \text { and } \forall s \in \mathbb{R} \\ G(x, s) \geq \gamma(x) & \text { for a.e. } x \in \Omega \text { and } \forall s \in \mathbb{R} ;\end{cases}
$$

an example of function $G$ satisfying the above assumptions is given by $G(x, s)=s^{4 / 3}$.

We will prove the following result.

Theorem 1. - Assume (2)-(6), then (1) admits a weak solution.

When $a_{i j}(x, u) \equiv \delta_{i j}$ this result is due to Rabinowitz (see [12, Theorem 4.12]); for the more general equation (1) we cannot apply directly the saddle point Theorem (see [12, Theorem 4.6]). Indeed, to find weak solutions of (1) we look for critical points of the functional $J: H_{0}^{1}(\Omega) \rightarrow \mathbb{R}$ defined by

$$
\begin{aligned}
& \forall u \in H_{0}^{1}(\Omega) \\
& J(u)=\frac{1}{2} \int_{\Omega} \sum_{i, j=1}^{n} a_{i j}(x, u) \mathrm{D}_{i} u \mathrm{D}_{j} u-\int_{\Omega} G(x, u)-\frac{1}{2} \int_{\Omega} b(x) u^{2} .
\end{aligned}
$$


As was noticed by Canino [3] the functional $J$ is not even locally Lipschitz continuous unless either the functions $a_{i j}(x, s)$ are independent of $s$ or $n=1$; therefore, to treat (1) a different notion of critical point is needed, see Section 2 where the nonsmooth critical point theory developed in [8] and [9] is briefly outlined. Such theory has already been used by Canino to find weak solutions of quasilinear elliptic equations; in her paper she studied equation (1) with $g(x, u)$ having a superlinear behaviour at infinity: under this assumption the functional satisfies the geometric requirements of the mountain pass theorem. In our case $g$ satisfies (6), that is we study a case of resonance, and a generalized saddle point theorem is required.

We point out that different problems involving quasilinear elliptic equations with coefficients depending on $u$ have been treated with variational methods by Arcoya and Boccardo [1] and by Struwe ([13], [14]).

An important tool to establish the geometrical properties of the functional $J$ is the following linear elliptic "operator at infinity".

$$
L^{\infty} u:=-\sum_{i, j=1}^{n} \mathrm{D}_{j}\left(A_{i j}(x) \mathrm{D}_{i} u\right)-b(x) u
$$

we denote by $\lambda_{j}(j \in \mathbb{N})$ its eigenvalues. Note that by (5) (and the same arguments used in the proof of Lemma 3 ) only a finite number of such eigenvalues are negative, $\lambda_{j} \rightarrow+\infty$ as $j \rightarrow \infty$ and the set of the corresponding eigenfunctions $e_{j}$ is a complete system in $H:=H_{0}^{1}(\Omega)$; in the sequel we denote by $\|\cdot\|$ the norm in $H$ (the Dirichlet norm). We study the resonance case, i.e., when $L^{\infty}$ is not invertible, as it seems to be more interesting: indeed for the corresponding semilinear equation, if $L^{\infty}$ admits an inverse, the existence of a weak solution follows from standard elliptic estimates and Schauder's fixed point theorem; the results we obtain can be easily extended to the case where 0 is not an eigenvalue of (7), see Remark 4 in Section 4.

If the coefficients of the equation satisfy the following symmetries

$$
\left\{\begin{array}{l}
a_{i j}(x, s)=a_{i j}(x,-s) \text { and } g(x, s)=-g(x,-s) \\
\forall s \in \mathbb{R} \text { and for a.e. } x \in \Omega
\end{array}\right.
$$

then index theory can be applied to prove the following multiplicity result. 
Theorem 2.- Assume (2)-(6) (with $\alpha \equiv 0$ ) and (8); define

$$
g_{0}(x):=\limsup _{s \rightarrow 0} \frac{g(x, s)}{s}
$$

and

$$
L^{0} u:=-\sum_{i, j=1}^{n} \mathrm{D}_{j}\left(a_{i j}(x, 0) \mathrm{D}_{i} u\right)-b(x) u-g_{0}(x) u .
$$

Let $k$ be the number of nonpositive eigenvalues of $L^{\infty}$ and let $m<+\infty$ be the number of nonpositive eigenvalues of $L^{0}$; if $k>m$, then equation (1) admits at least $k-m$ pairs of weak solutions.

Remark 1. - If $g(x, 0)=0$ for a.e. $x \in \Omega$, then $u \equiv 0$ is a solution of (1). By an extension of Morse theory to continuous functionals recently introduced by Corvellec [6] and with the methods used in [7] it can be proved that if $k \neq m$ ( $k$ and $m$ as in Theorem 2), then (1) admits a nontrivial solution.

Remark 2. - If in (5) and (6) we assume that $b, \alpha, \beta \in L^{p}(\Omega)$ for some $p>n / 2$, then all weak solutions of (1) are in $L^{\infty}(\Omega)$ (see e.g. [4]). Consequently, further regularity informations can be obtained by the techniques of [11].

This paper is organized as follows.

In Section 2 we prove a generalization to continuous functionals of the saddle point theorem and of a multiplicity result: this is done in the framework of the nonsmooth critical point theory introduced by Degiovanni et al. and with a weaker version of the Palais-Smale condition introduced by Cerami [5]. In Section 3, we prove some technical results concerning the properties of the functional $J$. Finally, in Section 4, we prove Theorems 1 and 2 .

\section{General results of nonsmooth critical point theory}

In this paper we deal with the critical point theory for continuous functionals developed in [8] and [9]: in this section we introduce the basic definitions and tools. 
Definition 1. - Let $(X, d)$ be a metric space, $I \in C(X, \mathbb{R})$ and let $x \in X$. We denote by $|d I|(x)$ the supremum of the $\sigma \in[0,+\infty]$ such that there exist $\delta>0$ and a continuous map

$$
\mathcal{H}: B(x, \delta) \times[0, \delta] \longrightarrow B(x, 2 \delta)
$$

such that $\forall y \in B(x, \delta), \forall t \in[0, \delta]$ we have

$$
d(\mathcal{H}(y, t), y) \leq t \quad \text { and } \quad I(\mathcal{H}(y, t)) \leq I(y)-\sigma t
$$

where $B(x, r):=\{y \in X \mid d(x, y)<r\} ;|d I|(x)$ is called the weak slope of $I$ at $x$.

This notion for continuous functionals has been independently introduced by Katriel [10].

DEFINITION 2.- Let $(X, d)$ be a metric space and $I \in C(X, \mathbb{R})$; a point $x \in X$ is said to be critical for $I$ if $|d I|(x)=0$. A real number $c$ is said to be a critical value for $I$ if there exists $x \in X$ such that $I(x)=c$ and $|d I|(x)=0$.

Let $(X, d)$ be a metric space, $I \in C(X, \mathbb{R})$ and $c \in \mathbb{R}$; we define the sets

$$
\begin{gathered}
I^{c}:=\{x \in X \mid I(x) \leq c\} \\
K:=\{x \in X .|| d I \mid(x)=0\}, \quad K_{c}:=\{x \in K \mid I(x)=c\}
\end{gathered}
$$

and

$$
B_{r}:=\{x \in X \mid d(x, 0) \leq r\}, \quad \partial B_{r}:=\{x \in X \mid d(x, 0)=r\} .
$$

Next we deal with Palais-Smale sequences (PS sequences): following [9] we say that a functional $I \in C(X, \mathbb{R})$ satisfies the PS condition if every sequence $\left\{x_{m}\right\} \subset X$ such that $I\left(x_{m}\right)$ is bounded and $|d I|\left(x_{m}\right) \rightarrow 0$ is relatively compact. We will prove that the functional $J$ satisfies a weaker version of the PS condition which is due to Cerami [5]: in our framework the Palais-Smale-Cerami (PSC) sequences and the PSC condition can be defined as follows. 
Definition 3.- Let $X$ be a Banach space and let $I \in C(X, \mathbb{R})$. We say that a sequence $\left\{x_{m}\right\} \subset X$ is a PSC sequence if

$$
\begin{gathered}
\exists k>0 \text { such that }\left|I\left(x_{m}\right)\right| \leq k \\
\left(1+\left\|x_{m}\right\|\right)|d I|\left(x_{m}\right) \longrightarrow 0 .
\end{gathered}
$$

We say that I satisfies the PSC condition if all its PSC sequences are precompact.

We generalize the saddle point theorem to the case of continuous functionals satisfying the PSC condition (see also Theorem 3.7 in [8]).

Theorem 3.- Let $X$ be a Banach space, $I: X \rightarrow \mathbb{R}$ a continuous functional, $(D, S)$ a compact pair and $\psi: S \rightarrow X$ a continuous map. Let $\Phi:=\{\varphi \in C(D, X)|\varphi| S=\psi\}$ and assume that there exists a closed subset $A$ of $X$ such that

$$
\begin{aligned}
& A \cap \psi(S)=\emptyset, \\
& \inf _{A} I \geq \max _{S}(I \circ \psi) \quad \text { and } \quad A \cap \varphi(D) \neq \emptyset, \quad \forall \varphi \in \Phi .
\end{aligned}
$$

If I satisfies the PSC condition and

$$
c:=\inf _{\Phi} \max _{D}(I(\varphi))
$$

then $K_{c} \neq \emptyset$; moreover, if $c=\inf _{A} I$, then $K_{c} \cap A \neq \emptyset$.

Proof. - If $I \in C^{0}(X, \mathbb{R})$ and $I$ satisfies PS the result is proved in [8] where $X$ is any complete metric space. If $I$ only satisfies PSC, then we can define a different metric $\tilde{d}$ on $X$ by

$$
\tilde{d}(x, y):=\int_{0}^{1} \frac{\|y-x\|}{2+\|x+\tau(y-x)\|} \mathrm{d} \tau=\int_{0}^{\|y-x\|} \frac{\mathrm{d} s}{2+\left\|x+s \frac{y-x}{\|y-x\|}\right\|}
$$

it is not difficult to verify that $(X, \widetilde{d})$ is a complete metric space and that the topology endowed by $\widetilde{d}$ is equal to the norm topology: furthermore if $|\widetilde{d} I|$ is the weak slope of $I$ with respect to the metric $\widetilde{d}$, then $|\widetilde{d} I|(x)=$ $(1+\|x\|)|d I|(x)$ therefore if $I$ satisfies PSC with respect to the norm metric, then it also satisfies PS with respect to the metric $\tilde{d}$ and the result follows. 
If the functional is even, then index theory applies.

THEOREM 4.- Let $X$ be a Banach space, $V \subset X$ a nontrivial finite dimensional subspace, $I: X \rightarrow \mathbb{R}$ a continuous functional satisfying the $P S C$ condition and $I(0)=0$; assume moreover that:

(i) I is even;

(ii) $\exists \rho, \sigma>0$ and a subspace $U$ of $X$ of finite codimension such that $I(x) \geq \sigma$ for all $x \in \partial B_{\rho} \cap U$ and $\operatorname{codim}(U)<\operatorname{dim}(V)$;

(iii) $\exists R>\rho$ such that if $x \in \partial B_{R} \cap V$, then $I(x) \leq 0$.

Then $I$ admits at least $\operatorname{dim}(V)-\operatorname{codim}(U)$ pairs of critical points.

Proof. - The proof follows the same lines of Theorem 9.12 in [12], see also [15]: we use the same notations as in [12] and give a sketch of the proof.

Let $k=\operatorname{dim}(V)$, let $\left\{e_{j}\right\}_{j=1, \ldots, k}$ be a basis of $V$ and for $m=1, \ldots, k$ let $E_{m}=\operatorname{span}\left\{e_{1}, \ldots, e_{m}\right\}$ and $\Gamma_{m}$ be the families of sets defined in [12]. We can define $k$ minimax levels $c_{m}$ of $J$ and prove that if $m>\operatorname{codim}(U)$, then $c_{m}>0$ and $c_{m}$ is a critical level of $J$ by a standard application of the deformation theorem. Moreover, if $c_{n}=c_{m}$ for $\operatorname{dim}(V) \geq n>m>$ $\operatorname{codim}(U)$ then $\gamma\left(K_{c_{m}}\right) \geq n-m+1$ and the set of critical points at level $c_{m}$ is infinite.

There are two differences between our case and [12]: we have $m=$ $1, \ldots, k$ instead of $m \in \mathbb{N}$ and the classical deformation lemma does not hold in the framework of nonsmooth critical point theory. On the other hand, an odd deformation is provided by Theorem 2.17 in [8] for functionals satisfying PS and the same observation as in the proof of Theorem 3 applies.

We introduce a notion of differentiability which is suitable for handling weak solutions of PDE's.

Definition 4. - Let $X$ be a Banach space, let $I \in C(X, \mathbb{R})$ and let $Y$ be a dense subspace of $X$. If the directional derivative of $I$ exists for all $x$ in $X$ in all the directions $y \in Y$ (i.e. $\forall x \in X, \forall y \in Y, \exists I^{\prime}(x)[y]$ ) we say that $I$ is weakly $Y$-differentiable and we call weak $Y$-slope in $x$ the extended real number

$$
\begin{gathered}
\left\|I_{Y}^{\prime}(x)\right\|:=\sup \left\{I^{\prime}(x)[y] \mid y \in Y,\|y\|_{X}=1\right\} . \\
-579-
\end{gathered}
$$




\section{Gianni Arioli and Filippo Gazzola}

We focus our attention on functionals $I$ defined on $H_{0}^{1}(\Omega)$ of the following type:

$$
I(u)=\int_{\Omega} L(x, u, \nabla u) \mathrm{d} x,
$$

where $\Omega \subset \mathbb{R}^{n}(n \geq 3)$ is open and bounded, and there exists $h \in[0,+\infty)$, $h_{1} \in L^{1}(\Omega), h_{2} \in L_{\text {loc }}^{1}(\Omega), h_{3} \in L_{\text {loc }}^{\infty}(\Omega)$ such that $L: \Omega \times \mathbb{R} \times \mathbb{R}^{n} \rightarrow \mathbb{R}$ satisfies:

$$
\left\{\begin{array}{l}
\forall(s, \xi) \in \mathbb{R} \times \mathbb{R}^{n}, \\
L(x, s, \xi) \text { is measurable with respect to } x \text { for a.e. } x \in \Omega \\
L(x, s, \xi) \text { is of class } C^{1} \text { with respect to }(s, \xi) \\
|L(x, s, \xi)| \leq h_{1}(x)+h\left(|s|^{2 n /(n-2)}+|\xi|^{2}\right) \\
\left|\frac{\partial L}{\partial s}(x, s, \xi)\right| \leq h_{2}(x)+h_{3}(x)\left(|s|^{2 n /(n-2)}+|\xi|^{2}\right) \\
\left|\frac{\partial L}{\partial \xi}(x, s, \xi)\right| \leq h_{2}(x)+h_{3}(x)\left(|s|^{2 n /(n-2)}+|\xi|^{2}\right) .
\end{array}\right.
$$

We obtain a lower estimate of the weak slope of $I$ by means of the weak $C_{c}^{\infty}(\Omega)$-slope; the following lemma is a reformulation of Theorem 1.5 in [3].

LEMMA 1. - Let I be as in (9), with $L$ as in (10), then $I \in C\left(H_{0}^{1}(\Omega), \mathbb{R}\right)$ and $I$ is weakly $Y$-differentiable with $Y=C_{c}^{\infty}(\Omega)$; furthermore, $\forall u \in$ $H_{0}^{1}(\Omega)$ we have

$$
|d I|(u) \geq \sup \left\{I^{\prime}(u)[v] \mid v \in C_{c}^{\infty}(\Omega),\|v\|_{H_{0}^{1}(\Omega)}=1\right\}=:\left\|I_{Y}^{\prime}(u)\right\| ;
$$

in particular if $u \in H_{0}^{1}(\Omega)$ is a critical point of $I$ (in the sense of Definition 2) then

$$
I^{\prime}(u)[v]=\int_{\Omega}\left(\frac{\partial L}{\partial \xi}(x, u, \nabla u) \nabla v+\frac{\partial L}{\partial s}(x, u, \nabla u) v\right)=0, \quad \forall v \in C_{c}^{\infty}(\Omega) .
$$

We can now state the version of the saddle point theorem which we use.

THEOREM 5.- Let $H_{0}^{1}(\Omega)=V \oplus W$, where $V \neq\{0\}$ is finite dimensional. Assume $L: \Omega \times \mathbb{R} \times \mathbb{R}^{n} \rightarrow \mathbb{R}$ satisfies (10), let $I$ be defined as in (9) and assume that: 
(i) if $\left\{u_{m}\right\} \subset H_{0}^{1}(\Omega)$ is a sequence such that $I\left(u_{m}\right)$ is bounded and

$$
\begin{gathered}
\left(1+\left\|u_{m}\right\|\right) \int_{\Omega}\left(\frac{\partial L}{\partial \xi}\left(x, u_{m}, \nabla u_{m}\right) \nabla v+\frac{\partial L}{\partial s}\left(x, u_{m}, \nabla u_{m}\right) v\right) \mathrm{d} x= \\
=\left\langle\Upsilon_{m}, v\right\rangle, \quad \forall v \in C_{c}^{\infty}(\Omega)
\end{gathered}
$$

with $\Upsilon_{m} \rightarrow 0$ in $H^{-1}(\Omega)$, then $\left\{u_{m}\right\}$ is precompact;

(ii) $\exists \beta \in \mathbb{R}$ such that $\forall x \in W$ we have $I(x) \geq \beta$;

(iii) $\exists \alpha<\beta$ and $\exists R>0$ such that if $x \in \partial B_{R} \cap V$, then $I(x) \leq \alpha$.

Then the quasilinear equation

$$
-\nabla \cdot\left(\frac{\partial L}{\partial \xi}(x, u, \nabla u)\right)+\frac{\partial L}{\partial s}(x, u, \nabla u)=0
$$

has a solution $u \in H_{0}^{1}(\Omega)$ in distributional sense.

Proof. - By assumption (i) and Lemma 1 the functional $I$ satisfies the PSC condition.

Let $D=B_{R} \cap V, S=\partial B_{R} \cap V$ and $\Phi=\{h \in C(D, X) \mid h=$ id on $S\}$. By (ii) and (iii) the assumptions of Theorem 3 are fulfilled, with $A=W$, therefore $I$ admits a critical value

$$
c=\inf _{h \in \Phi} \max _{x \in D} I(h(x)) \geq \beta
$$

If the equation is invariant under a $\mathbb{Z}_{2}$-action, then we can rephrase Theorem 4 as follows.

THEOREM 6. - Take the same assumptions of Theorem 5 and assume moreover that $I$ is even and that there exists $\rho \in(0, R)$ and a subspace $U$ of $H_{0}^{1}(\Omega)$ of finite codimension such that:

(iv) $I(x) \geq \beta$ for all $x \in \partial B_{\rho} \cap U$;

(v) $\operatorname{codim}(U)<\operatorname{dim}(V)$.

Then the quasilinear equation

$$
-\nabla \cdot\left(\frac{\partial L}{\partial \xi}(x, u, \nabla u)\right)+\frac{\partial L}{\partial s}(x, u, \nabla u)=0
$$

admits at least $\operatorname{dim}(V)-\operatorname{codim}(U)$ pairs of distinct solutions in distributional sense. 


\section{Properties of the functional $J$}

We wish now to adapt the previous result to the functional $J$. Consider the operator $L: \Omega \times \mathbb{R} \times \mathbb{R}^{n} \rightarrow \mathbb{R}$ defined by

$$
L(x, s, \xi)=\frac{1}{2} \sum_{i, j=1}^{n} a_{i j}(x, s) \xi_{i} \xi_{j}-G(x, s)-\frac{1}{2} b(x) s^{2}
$$

it is not difficult to see that $L$ satisfies conditions (10) and Lemma 1 applies.

Some remarks about assumptions (6) are in order: in [3] the assumption about $g$ is that of superlinear growth at infinity and this leads to the usual estimates of superquadratic functionals; in [12] it is assumed that $g$ is bounded while in our case (6) allows a strict sublinear growth but avoids the possibility of $G(x, s)$ going to $-\infty$ as $|s| \rightarrow \infty$. There are two reasons for our assumptions (6): the first one is to prove Lemma 4, the second one is used in Proposition 1 and is explained in the following.

Lemma 2.- Assume (6), and let $\left\{u_{m}\right\} \subset H$ be a sequence such that $\left\|u_{m}\right\| \rightarrow \infty$; then, as $m \rightarrow \infty$,

$$
\left|\int_{\Omega} G\left(x, u_{m}\right)\right|=\mathrm{o}\left(\left\|u_{m}\right\|^{2}\right) .
$$

Proof. - Let $\left\{u_{m}\right\} \subset H$ be such that $\left\|u_{m}\right\| \rightarrow \infty$; we claim that there exists a sequence $\left\{\varepsilon_{m}\right\} \subset \mathbb{R}^{+}$such that $\varepsilon_{m} \rightarrow 0$ and, for a.e. $x \in \Omega$,

$$
\left|G\left(x, u_{m}(x)\right)\right| \leq\left\|u_{m}\right\|^{1 / 2} \cdot \alpha(x)+\left\|u_{m}\right\| \cdot \frac{\beta(x)}{2}+\varepsilon_{m}\left|u_{m}(x)\right|^{2} .
$$

We prove (11) in the case $u_{m}(x)>0$, the case $u_{m}(x)<0$ being similar. If $u_{m}(x)<\left\|u_{m}\right\|^{1 / 2}$ then, by (6) we have

$$
\begin{aligned}
\left|G\left(x, u_{m}(x)\right)\right| & \leq \int_{0}^{\left\|u_{m}\right\|^{1 / 2}}|g(x, t)| \mathrm{d} t \\
& \leq \int_{0}^{\left\|u_{m}\right\|^{1 / 2}}(\alpha(x)+\beta(x) t) \mathrm{d} t \\
& \leq\left\|u_{m}\right\|^{1 / 2} \alpha(x)+\left\|u_{m}\right\| \frac{\beta(x)}{2}
\end{aligned}
$$


Weak solutions of quasilinear elliptic PDÉ's at resonance

and (11) follows. If $u_{m}(x) \geq\left\|u_{m}\right\|^{1 / 2}$, note that by applying Hölder inequality and by $(6)$ we get

$$
\begin{aligned}
\int_{\left\|u_{m}\right\|^{1 / 2}}^{u_{m}(x)} t\left|\frac{g(x, t)}{t}\right| \mathrm{d} t & \leq\left(\int_{\left\|u_{m}\right\|^{1 / 2}}^{u_{m}(x)} t^{2} \mathrm{~d} t\right)^{1 / 2}\left(\int_{\left\|u_{m}\right\|^{1 / 2}}^{u_{m}(x)}\left|\frac{g(x, t)}{t}\right|^{2} \mathrm{~d} t\right)^{1 / 2} \\
& \leq\left|u_{m}(x)\right|^{3 / 2} \varepsilon_{m}\left|u_{m}(x)\right|^{1 / 2}
\end{aligned}
$$

by (12) we have

$$
\left|G\left(x, u_{m}(x)\right)\right|<\left\|u_{m}\right\|^{1 / 2} \alpha(x)+\left\|u_{m}\right\| \frac{\beta(x)}{2}+\int_{\left\|u_{m}\right\|^{1 / 2}}^{u_{m}(x)} t\left|\frac{g(x, t)}{t}\right| \mathrm{d} t
$$

and (11) follows.

To complete the proof note that, by integrating (11) we have

$$
\left|\int_{\Omega} G\left(x, u_{m}\right)\right| \leq c\left\|u_{m}\right\|+\varepsilon_{m}\left\|u_{m}\right\|_{2}^{2}
$$

With the next result we prove that for every unbounded sequence $\left\{u_{m}\right\} \subset H$ such that $J\left(u_{m}\right)$ is upper bounded we can estimate the growth of its $H$-norm by means of its $L^{2}$-norm.

Lemma 3.- There exists $\eta>0$ such that if $\left\{u_{m}\right\} \subset H$ is a sequence such that $J\left(u_{m}\right)$ is upper bounded and $\left\|u_{m}\right\| \rightarrow \infty$ as $m \rightarrow \infty$, then

$$
\left\|u_{m}\right\| \leq \eta\left\|u_{m}\right\|_{2}
$$

Proof. - By (5), for all $\varepsilon>0$ there exist $b_{1} \in L^{n / 2}$ and $b_{2} \in L^{\infty}$ such that $b=b_{1}+b_{2}$ and $\left\|b_{1}\right\|_{n / 2}<\varepsilon$. By (2) we have

$$
J\left(u_{m}\right) \geq c\left\|u_{m}\right\|^{2}-\int_{\Omega} G\left(x, u_{m}\right)-\frac{1}{2} \int_{\Omega} b u_{m}^{2}
$$

but, by Lemma 2 ,

$$
\left\|u_{m}\right\|^{-2}\left|\int_{\Omega} G\left(x, u_{m}\right)\right| \longrightarrow 0
$$


and, since

$$
\begin{aligned}
\left|\int_{\Omega} b u_{m}^{2}\right| & \leq \int_{\Omega}\left|b_{1} u_{m}^{2}\right|+\int_{\Omega}\left|b_{2} u_{m}^{2}\right| \\
& \leq\left\|b_{1}\right\|_{n / 2}\left\|u_{m}^{2}\right\|_{n /(n-2)}+\left\|b_{2}\right\|_{\infty}\left\|u_{m}\right\|_{2}^{2},
\end{aligned}
$$

the result follows by choosing $\varepsilon$ small enough and taking into account that $H$ is continuously embedded into $L^{2 n /(n-2)}$.

Remark 3. - Using (3) and a result of [2] it has been proved (see [3, Theorem 2.1]) that it makes sense to evaluate $J^{\prime}(u)$ in $u$ because all the integrals involved in $J^{\prime}(u)[u]$ exist.

We wish to prove that the assumption (i) of Theorem 5 holds; we achieve this task in two steps: we first prove that a PSC sequence is bounded.

LEMma 4. - All the PSC sequences for $J$ are bounded.

Proof. - Assume the converse, then, by Lemma 1, there exist $k>0$ and a sequence $\left\{u_{m}\right\} \subset H$ such that

$$
\left\{\begin{array}{l}
\left\|u_{m}\right\| \longrightarrow \infty \\
\left\|J_{Y}^{\prime}\left(u_{m}\right)\right\| \cdot\left\|u_{m}\right\| \longrightarrow 0 \\
\left|J\left(u_{m}\right)\right|<k
\end{array}\right.
$$

By Remark 3 we can evaluate

$$
\begin{aligned}
J^{\prime}\left(u_{m}\right)\left[u_{m}\right]= & \int_{\Omega} \sum_{i, j=1}^{n} a_{i j}\left(x, u_{m}\right) \mathrm{D}_{i} u_{m} \mathrm{D}_{j} u_{m}-\int_{\Omega} g\left(x, u_{m}\right) u_{m}+ \\
& +\frac{1}{2} \int_{\Omega} \sum_{i, j=1}^{n} \frac{\partial a_{i j}}{\partial s}\left(x, u_{m}\right) \mathrm{D}_{i} u_{m} \mathrm{D}_{j} u_{m} u_{m}-\int_{\Omega} b(x) u_{m}^{2}
\end{aligned}
$$


from the boundedness of $J\left(u_{m}\right)$ we know that

$$
\begin{aligned}
\int_{\Omega} \sum_{i, j=1}^{n} a_{i j}\left(x, u_{m}\right) \mathrm{D}_{i} u_{m} \mathrm{D}_{j} u_{m} & -\int_{\Omega} b(x) u_{m}^{2}= \\
= & 2 \int_{\Omega} G\left(x, u_{m}\right)+\mathrm{O}(1) \text { as } m \rightarrow \infty ;
\end{aligned}
$$

hence, if $m \rightarrow \infty$, by (13) and the Hahn-Banach Theorem we have

$$
\begin{aligned}
& 2 \int_{\Omega} G\left(x, u_{m}\right)-\int_{\Omega} g\left(x, u_{m}\right) u_{m}+ \\
& \quad+\frac{1}{2} \int_{\Omega} \sum_{i, j=1}^{n} \frac{\partial a_{i j}}{\partial s}\left(x, u_{m}\right) \mathrm{D}_{i} u_{m} \mathrm{D}_{j} u_{m} u_{m}=\mathrm{O}(1) .
\end{aligned}
$$

Define $v_{m}(x):=u_{m}(x) /\left\|u_{m}\right\|$, then, $\left\|v_{m}\right\| \equiv 1$ and $\exists v \in H$ such that on a subsequence $v_{m} \rightarrow v$ in $L^{2}$ and pointwise a.e. in $\Omega$; by Lemma 3 we know that $v \neq 0$.

By (6) we infer that $2 G\left(x, u_{m}\right)-g\left(x, u_{m}\right) u_{m} \rightarrow+\infty$ on a subset of $\Omega$ with positive measure, hence by Fatou's lemma $\int_{\Omega}\left(2 G\left(x, u_{m}\right)-g\left(x, u_{m}\right) u_{m}\right) \rightarrow$ $+\infty$. Furthermore, by (3) we have

$$
\int_{\Omega} \sum_{i, j=1}^{n} \frac{\partial a_{i j}}{\partial s}\left(x, u_{m}\right) \mathrm{D}_{i} u_{m} \mathrm{D}_{j} u_{m} u_{m} \geq 0,
$$

and the left hand side of (14) diverges to $+\infty$, contradiction.

We can now prove that the functional $J$ satisfies the PSC condition.

LEMma 5. - Let $\left\{u_{m}\right\}$ be a PSC sequence for the functional $J$, then $\left\{u_{m}\right\}$ is precompact.

Proof. - Let $\left\{u_{m}\right\}$ be a PSC sequence; by Lemma $4\left\{u_{m}\right\}$ is a bounded PS sequence, hence $u_{m}-u$ and $b u_{m} \rightarrow b u$ in $H^{-1}(\Omega)$ on a subsequence by a standard procedure (see e.g. [4]). Analogously, up to a subsequence, we get $g\left(x, u_{m}\right) \rightarrow g(x, u)$ in $H^{-1}(\Omega)$. The result then follows from Lemma 2.3 and Corollary 1.8 in [3]. 


\section{Proofs of the results}

Assume that $\lambda_{k}=0$ (eigenvalue relative to (7)) and consider the spaces

$$
H_{k}^{+}:=\overline{\operatorname{span}\left\{e_{j} \mid \lambda_{j}>0\right\}}, \quad H_{k}^{-}:=\operatorname{span}\left\{e_{j} \mid \lambda_{j} \leq 0\right\}
$$

we have $H=H_{k}^{+} \oplus H_{k}^{-}$. For all $u \in H$ we denote by $u^{k+}$ (respectively $u^{k-}$ ) its component over $H_{k}^{+}$(respectively $H_{k}^{-}$).

We prove that the geometrical requirements of the saddle point theorem hold.

Proposition 1.- Assume (4), (5) and (6), then

(i) $\exists \beta \in \mathbb{R}$ such that $\forall u \in H_{k}^{+}$we have $J(u) \geq \beta$;

(ii) $\exists \alpha<\beta$ and $\exists R>0$ such that if $u \in H_{k}^{-}$and $\|u\|=R$, then $J(u) \leq \alpha$.

Proof. - Statement (i) is proved if we prove that for every sequence $\left\{u_{m}\right\} \subset H_{k}^{+}$such that $\left\|u_{m}\right\| \rightarrow \infty$ we have $J\left(u_{m}\right) \rightarrow+\infty$; for such a sequence $\left\{u_{m}\right\}$, by Lemma 2 we know that $\int_{\Omega} G\left(x, u_{m}\right) /\left\|u_{m}\right\|^{2} \rightarrow 0$, therefore it suffices to prove that for $m$ large enough

$$
\int_{\Omega} \sum_{i, j=1}^{n} a_{i j}\left(x, u_{m}\right) \mathrm{D}_{i} v_{m} \mathrm{D}_{j} v_{m}-\int_{\Omega} b v_{m}^{2} \geq c>0,
$$

where $v_{m}:=u_{m} /\left\|u_{m}\right\|$. There exists $v \in H(\|v\| \leq 1)$ such that $v_{m} \rightarrow v$ on a subsequence; as $b \in L^{n / 2}$, on a subsubsequence $\int_{\Omega} b v_{m}^{2} \rightarrow \int_{\Omega} b v^{2}$.

To conclude the proof of (i) we use the same device as in [7].

Let

$$
\ell_{m}=\int_{\Omega} \sum_{i, j=1}^{n} a_{i j}\left(x, u_{m}\right) \mathrm{D}_{i} v_{m} \mathrm{D}_{j} v_{m}
$$

as $\left\{\ell_{m}\right\}$ is bounded, on a subsequence $\ell_{m} \rightarrow \ell$ and two cases may occur: 
Weak solutions of quasilinear elliptic PDE's at resonance

1) $\ell>\int_{\Omega} \sum_{i, j=1}^{n} A_{i j}(x) \mathrm{D}_{i} v \mathrm{D}_{j} v$; in this case, as $v \in H_{k}^{+}$, (15) follows;

2) $\ell \leq \int_{\Omega} \sum_{i, j=1}^{n} A_{i j}(x) \mathrm{D}_{i} v \mathrm{D}_{j} v$; then $v_{m} \rightarrow v$ strongly in $H$, because by (2)

$$
\begin{aligned}
c\left\|v_{m}-v\right\|^{2} \leq & \int_{\Omega} \sum_{i, j=1}^{n} a_{i j}\left(x, u_{m}\right) \mathrm{D}_{i}\left(v_{m}-v\right) \mathrm{D}_{j}\left(v_{m}-v\right)= \\
= & \int_{\Omega} \sum_{i, j=1}^{n} a_{i j}\left(x, u_{m}\right) \mathrm{D}_{i} v_{m} \mathrm{D}_{j} v_{m}+ \\
& -2 \int_{\Omega} \sum_{i, j=1}^{n} a_{i j}\left(x, u_{m}\right) \mathrm{D}_{i} v_{m} \mathrm{D}_{j} v+ \\
& +\int_{\Omega} \sum_{i, j=1}^{n} a_{i j}\left(x, u_{m}\right) \mathrm{D}_{i} v \mathrm{D}_{j} v ;
\end{aligned}
$$

but $\mathrm{D}_{i} v_{m} \rightarrow \mathrm{D}_{i} v$ in $L^{2}$ and $a_{i j}\left(x, u_{m}\right) \mathrm{D}_{j} v \rightarrow A_{i j}(x) \mathrm{D}_{j} v$ in $L^{2}$ by Lebesgue's dominated convergence theorem, therefore

$$
\int_{\Omega} a_{i j}\left(x, u_{m}\right) \mathrm{D}_{i} v_{m} \mathrm{D}_{j} v \longrightarrow \int_{\Omega} A_{i j}(x) \mathrm{D}_{i} v \mathrm{D}_{j} v
$$

and

$$
\int_{\Omega} a_{i j}\left(x, u_{m}\right) \mathrm{D}_{i} v \mathrm{D}_{j} v \longrightarrow \int_{\Omega} A_{i j}(x) \mathrm{D}_{i} v \mathrm{D}_{j} v
$$

so $v_{m} \rightarrow v$ in $H$ and the claim follows.

To prove (ii) it suffices to prove that if $\left\{u_{m}\right\} \subset H_{k}^{-}$is a sequence such that $\left\|u_{m}\right\| \rightarrow \infty$, then $J\left(u_{m}\right) \rightarrow-\infty$. Recall that $\operatorname{dim} H_{k}^{-}=k$; hence, for such a sequence $\left\{u_{m}\right\}$, by (6) we know that $G\left(x, u_{m}\right) \rightarrow+\infty$ on a subset of $\Omega$ with positive measure, and by Fatou's lemma

$$
\int_{\Omega} G\left(x, u_{m}\right) \longrightarrow+\infty
$$

the result follows taking into account that if $u_{m} \in H_{k}^{-}$, then the quadratic part of the functional is negative. 


\section{Gianni Arioli and Filippo Gazzola}

By Lemma 5 and the above proposition, the assumptions of Theorem 5 are fulfilled and Theorem 1 is proved.

To prove Theorem 2, consider that by the definition of the operator $L^{0}$ there exists a subspace $U \subset H$ of codimension $m$ such that $\left(L^{0} u, u\right)>0$ for all $u \in U$. By the semipositivity condition (3) we infer that

$$
\begin{aligned}
J(u) & \geq \frac{1}{2} \int_{\Omega} \sum_{i, j=1}^{n} a_{i j}(x, 0) \mathrm{D}_{i} u \mathrm{D}_{j} u-\frac{1}{2} \int_{\Omega} b(x) u^{2}-\frac{1}{2} \int_{\Omega} \psi(x, u) u^{2} \\
& \geq \frac{1}{2}\left(L^{0} u, u\right)
\end{aligned}
$$

where

$$
\psi(x, s)= \begin{cases}\max \left(\frac{2 G(x, s)}{s^{2}}, g_{0}(x)\right) & \text { if } s \neq 0 \\ 0 & \text { if } s=0\end{cases}
$$

this proves that

$$
\lim _{u \rightarrow 0} \inf _{u \in U} \frac{J(u)}{\|u\|^{2}}>0
$$

and therefore there exist $\rho, \sigma>0$ such that the hypotheses of Theorem 6 are fulfilled. The proof of Theorem 2 then follows.

Remark 4. - If $L^{\infty}$ in (7) is invertible, then the existence of a weak solution $u$ and (1) follows with minor modifications of the proof.

\section{Acknowledgements}

The authors are very grateful to Marco Degiovanni for suggesting the problem and for many useful discussions. The second author has been supported by G.N.A.F.A.

\section{References}

[1] ARCOYA (D.) and BOCCARDO (L.) .- Critical points for multiple integrals of the calculus of variations, Arch. Rat. Mech. Anal. 134 (1996), pp. 249-274.

[2] Brezis (H.) and Browder (F. E.) .- Sur une propriété des espaces de Sobolev, C. R. Acad. Sc., Paris, 287 (1978), pp. A113-A115.

[3] CANino (A.) . - Multiplicity of solutions for quasilinear elliptic equations, Top. Meth. Nonlin. Anal. 6 (1995), pp. 357-370. 
[4] Canino (A.) and Degiovanni (M.) .- Nonsmooth critical point theory and quasilinear elliptic equations, Topological Methods in Differential Equations and Inclusions, Montreal 1994, NATO ASI Series, pp. 1-50.

[5] Cerami (G.) .- Un criterio di esistenza per i punti critici su varietà illimitate, Rend. Ist. Sci. Lombardo 112 (1978), pp. 332-336.

[6] Corvellec (J. N.) . - Morse theory for continuous functionals, to appear in J. Math. Anal. Appl. 196 (1995), pp. 1050-1072.

[7] Corvellec (J. N.) and Degiovanni (M.) .- Nontrivial solutions of quasilinear equations via nonsmooth Morse theory, J. Diff. Eq. 136 (1997), pp. 268-293.

[8] Corvellec (J. N.), Degiovanni (M.) and Marzocchi (M.) .- Deformation properties for continuous functionals and critical point theory, Top. Meth. Nonlin. Anal. 1 (1993), pp. 151-171.

[9] Degiovanni (M.) and Marzocchi (M.) . - A critical point theory for nonsmooth functionals, Ann. Mat. Pura Appl. IV, CLXVII (1994), pp. 73-100.

[10] KATRIEL (G.) .- Mountain pass theorems and global homeomorphism theorems, Ann. Inst. H.-Poincaré, A.N.L. 11 (1994), pp. 189-209.

[11] Ladyzhenskaya (O. A.) and Ural'tseva (N. N.) . - Linear and quasilinear elliptic equations, Academic Press, New York, 1968.

[12] RABINOWITZ (P. H.) .- Minimax methods in critical point theory with applications to differential equations, CBMS Reg. Conf. Series Math. 65, Amer. Math. Soc., Providence, R.I., 1986.

[13] STRUWE (M.) . - Multiple solutions of differential equations without the PalaisSmale condition, Math. Ann. 261 (1982), pp. 399-412.

[14] StRUWe (M.) .- Quasilinear elliptic eigenvalue problems, Comment. Math. Helvetici 58 (1983), pp. 509-527.

[15] SzULKIN (A.) .- Minimax principles for lower semicontinuous fonctions and applications to nonlinear boundary value problems, Ann. Inst. H.-Poincaré, A.N.L. 3 (1986), pp. 77-109. 